\title{
Production d'électrons relativistes par interaction laser-plasma et application à la génération d'impulsions femtosecondes d'X dans le domaine du keV
}

\author{
J.-R. Marquès, P.-G. David, J. Faure, S. Fritzler ${ }^{1}$, V. Malka1 , Z. Najmudin ${ }^{2}$, \\ A. Rousse ${ }^{1}$, K. Ta Phuoc ${ }^{1}$ et B. Walton ${ }^{2}$
}

Laboratoire d'Utilisation des Lasers Intenses, UMR 7605 du CNRS, CEA, École Polytechnique, Université Paris VI, 91128 Palaiseau cedex, France

${ }^{1}$ Laboratoire d'Optique Appliquée, UMR 7639 du CNRS, ENSTA, École Polytechnique, Chemin de la Hunière, 91761 Palaiseau, France

${ }^{2}$ Blackett Laboratory, Imperial College, London SW7 A2Z, U.K.

Résumé : Nous avons étudié la production de particules énergétiques par l'interaction d'un laser ultra-bref et intense avec un plasma issu de l'ionisation laser d'un jet de gaz. A haute densité électronique $\left(n_{e}>10^{99} \mathrm{~cm}^{-3}\right)$ un faisceau d'électrons relativistes est produit par sillage laser auto-modulé. A basse densité plasma $\left(n_{e}<10^{19} \mathrm{~cm}^{-3}\right)$ un faisceau de rayons $\mathrm{X}$ est produit par émission Larmor des électrons du plasma oscillants dans le champ laser. Nous avons étudié les distributions en énergies et en angles de ces deux de faisceaux de particules. Les distributions angulaires sont gaussiennes et orientées suivant l'axe laser. La distribution en énergie du faisceau d'X est large, piquée autour de $0.15 \mathrm{keV}$, et s'étend jusqu'à $2 \mathrm{keV}$. Celle du faisceau d'électrons est maxwellienne de température $T_{e} \approx 10 \mathrm{eV}$. Ces résultats ont été obtenus avec un laser $10 \mathrm{~Hz}$, ouvrant la porte à de nombreuses applications.

\section{PRODUCTION D'UN FAISCEAU D'ELECTRONS PAR SILLAGE LASER AUTOMODULE}

Dans le cadre des recherches sur la génération de champs électriques ultra-intenses, des électrons ont été accélérés en quelques centaines de microns à des énergies allant jusqu'à la centaine de MeV [1]. Ces expériences de démonstration utilisaient des lasers à faible cadence de tir. Nous avons conduit une série d'expériences [2] visant à mieux caractériser le faisceau d'électrons et à démontrer qu'une telle source d'électrons relativistes peut être produite à une cadence d'au moins $10 \mathrm{~Hz}$, devenant alors un outil intéressant pour diverses applications. La courte durée et le fort courant du faisceau pourrait par exemple créer des impulsions $\mathrm{X}$ ou $\gamma$ plus brèves et plus intenses que celles issues des accélérateurs actuels, utiles non seulement en physique, mais aussi pour l'étude de phénomènes rapides en biologie ou en chimie.

Le principe de l'accélération par sillage laser auto-modulé est le suivant : une impulsion laser se propage dans un plasma et excite (soit par instabilité Raman, soit par la force pondéromotrice) une onde plasma électronique. Si la durée de l'impulsion contient plusieurs période plasma, les gradients associés à l'onde plasma vont créer dans l'enveloppe du laser une modulation à la période plasma. Les gradients associés à cette modulation étant par conception résonants avec l'onde plasma, cette dernière va croître plus efficacement et induire une modulation encore plus forte. Cette instabilité d'auto-modulation s'arrête lorsque l'amplitude de l'onde plasma atteint le seuil de déferlement. Des électrons de l'onde plasma voient alors le très fort champ électrostatique de l'onde (pouvant dépasser $100 \mathrm{GV} / \mathrm{m}$ ) et peuvent être accélérés à des énergies pouvant atteindre $100 \mathrm{MeV}$ [1], voir dépasser le GeV.

\subsection{Dispositif expérimental}

L'expérience d'accélération d'électrons par sillage auto-modulé est schématisée en figure 1. Le laser $10 \mathrm{~Hz}$ - $30 \mathrm{TW}$ du Laboratoire d'Optique Appliquée $(\lambda=820 \mathrm{~nm}, F W H M=30 \mathrm{fs})$ était focalisé par une parabole hors-axe dans une tache de $6 \mu \mathrm{m}$ de rayon, conduisant à une intensité de $2 \times 10^{19} \mathrm{~W} / \mathrm{cm}^{2}$. Le plasma était créé par l'ionisation laser d'un jet supersonique de gaz d'hélium. Deux types de buses ont été utilisées, permettant de couvrir une gamme de densités électroniques allant de $10^{18}$ à $10^{20} \mathrm{e} / \mathrm{cm}^{3}$. Un faisceau sonde non-focalisé $(\phi=30 \mathrm{~mm}, \lambda=820 \mathrm{~nm}, F W H M=30 \mathrm{fs})$ couplé à un diagnostic d'imagerie à $90^{\circ}$ de l'axe laser permettait d'étudier le plasma et la propagation du laser principal (autofocalisation relativiste, réfraction, filamentation). Un intégrateur de charges placé juste après le jet de gaz mesurait le nombre 
total d'électrons accélérés. Un spectromètre à électrons couplé à des diodes mesurait le spectre en énergie des électrons dans la gamme $1 \mathrm{MeV}$ à $200 \mathrm{MeV}$. Un scintillateur amovible (cristal de YAG:Ce) imagé sur une caméra $\mathrm{CCD}$ ou des films dosimètres permettaient de mesurer le profil transverse du faisceau d'électrons.

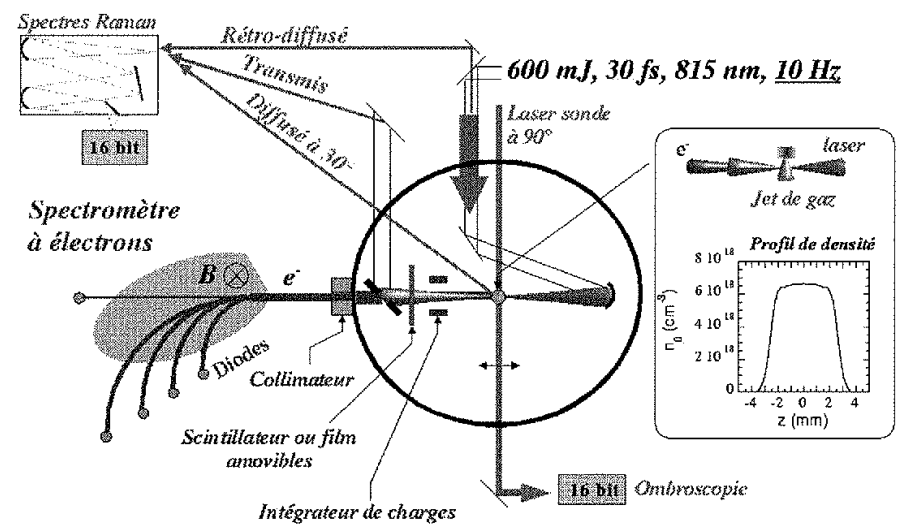

Figure 1. : Dispositif expérimental de l'expérience d'accélération d'électrons par sillage auto-modulé

\subsection{Résultats expérimentaux}

La distribution en énergie des électrons accélérés suivant l'axe laser a été mesurée pour de nombreux paramètres expérimentaux. Le graphe de gauche de la figure 2 montre des distributions obtenues à différentes pressions du jet de gaz (différentes densités électroniques $n_{e}$ du plasma). La distribution est maxwellienne et la température qui lui est associée est d'autant plus élevée que la densité plasma est plus faible (cf graphe de droite de la figure 2). Ce comportement est en accord avec le mécanisme d'accélération par une onde plasma, la vitesse de phase de l'onde augmentant quand $n_{e}$ diminue [3].

Le nombre et l'énergie (température) des électrons augmentent avec la puissance laser. Le nombre total d'électrons atteignait presque $10 \mathrm{nC}$. Le flux d'électrons d'énergies $>10 \mathrm{MeV}$ et accélérés suivant l'axe laser était d'environ $10^{10} \mathrm{e} / \mathrm{sr}$.
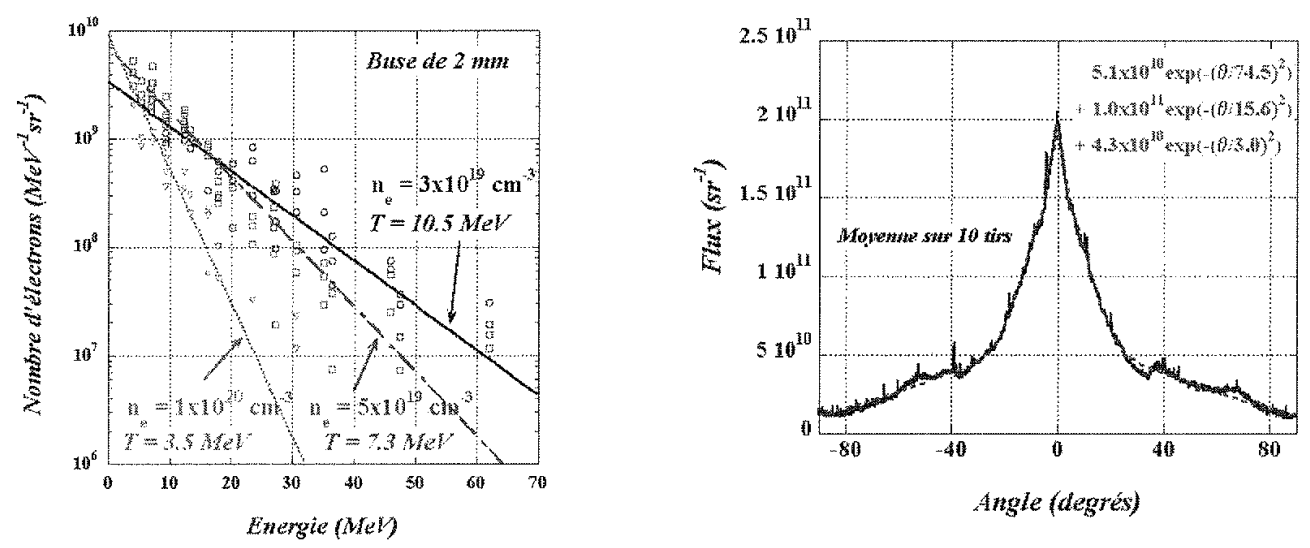

Figure. 2 : Gauche : distributions en énergie des électrons, pour une densité plasma de $n_{c}=3 \times 10^{19}$ (ronds), $5 \times 10^{19}$ (carrés), et $1.5 \times 10^{20} \mathrm{~cm}^{-3}$ (triangles). Les droites sont des interpolations de la forme $N e^{E / T}$, où $T$ est la température du spectre.

Droite : distribution angulaire du flux d'électrons déduite de films radiochromiques.

Le profil transverse (angulaire) du faisceau d'électrons est représenté sur le graphe de droite de la. 
figure 2. Il correspond à l'ensemble des électrons, toutes énergies confondues. La distribution semble constituée d'une composante de grande divergence $\left(\sigma \approx 50^{\circ}\right)$ et d'une ou deux composantes de plus faibles divergences $\left(\sigma_{\theta} \approx 11\right.$ et $2^{\circ}$ ). En supposant que les électrons sont produits dans la tache focale du laser $\left(\sigma_{\perp}\right.$ $\approx 3 \mu \mathrm{m}$ ), l'émittence est donc comprise entre $\varepsilon \approx 0.1$ à $0.6 \mathrm{~mm} . \mathrm{mrad}$. Notons enfin que des mesures faites avec des films radiochromiques intercalés entre des plaques de métal ont montré que les électrons sont d'autant plus collimatés que leur énergie est élevée.

\section{GENERATION D'UNE SOURCE X ULTRABREVE ET POLYCHROMATIQUE}

Avec l'arrivée des lasers ultra-intenses $\left(>10^{18} \mathrm{~W} / \mathrm{cm}^{2}\right)$, une nouvelle source ultra-brève d'X est possible. Elle exploite le rayonnement de Larmor produit par les électrons d'un plasma oscillants dans le champ électromagnétique d'un laser [4]. Ce rayonnement est simplement celui émit par un électron qui subit une accélération [5], celle induite par le champ laser dans le cas présent. Le spectre d'émission est large, continu, et s'étend à d'autant plus haute énergie que l'intensité laser est élevée. La durée d'émission est de l'ordre ou inférieure à la durée du laser. Cette source a pour principaux avantages d'être très brève (dizaines de fs), énergétique (keV), et directive.

\subsection{Dispositif de l'expérience de source $X$ par rayonnement de Larmor}

Le dispositif de cette expérience est schématisé en figure 3 . Un laser intense $(0.8 \mathrm{~J}, 30 \mathrm{fs})$ était focalisé sur le bord d'un jet de gaz. La densité du plasma créé était ajustée en changeant la pression en amont du jet et était maintenue en dessous du seuil d'excitation du sillage auto-modulé, évitant ainsi l'éjection d'électrons susceptible de créer des X parasites. Pour être sûr que de tels électrons ne viennent pas créer des $\mathrm{X}$ parasites suivant l'axe laser, des aimants permanents placés juste après le jet de gaz déviaient d'éventuels électrons accélérés.
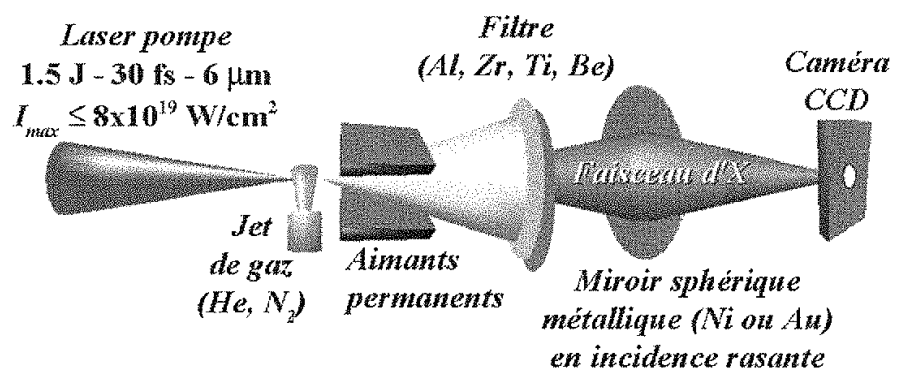

Figure 3. Dispositif expérimental de l'expérience de source X par rayonnement de Larmor dans le champ laser.

Le faisceau d'X produit était collecté par un miroir sphérique et focalisé sur une caméra CCD. La sélection en énergie des $\mathrm{X}$ collectés se faisait avec quatre types de filtres métalliques. Pour mesurer la distribution angulaire des $X$, les aimants permanents, le filtre métallique, le miroir sphérique, et la caméra CCD étaient fixés sur un bras pouvant pivoter autour d'un axe vertical passant par le foyer laser. Pour pouvoir changer la polarisation du laser, deux lames quart-d'onde étaient placées avant la parabole horsaxe.

\subsection{Résultats et discussion sur l'expérience de rayonnement de Larmor dans le champ laser}

La distribution angulaire des $\mathrm{X}$ d'énergie située autour de $350 \mathrm{eV}$ (filtre de titane) est présentée en figure 4 (graphe de gauche). Trois types de polarisation ont été étudiées : linéaire horizontale, linéaire verticale, et circulaire. Dans tous les cas la distribution est maximale dans la direction du faisceau laser et décrôit continûment jusqu'à au moins $40^{\circ}$. On voit que la divergence ne semble pas dépendre du type de polarisation. Le nombre $\mathrm{d}^{\prime} \mathrm{X}$ est par contre bien plus important en polarisation circulaire. Ce comportement a été observé quelque soit l'énergie des $X$ et est en bon accord avec le fait qu'en 
polarisation circulaire l'électron subit une accélération maximale en continu.

Le graphe de droite de la figure 4 présente la distribution en énergie (suivant l'axe laser). Elle est large, présente un maximum autour de $0.15 \mathrm{keV}$, et s'étend jusqu'à au moins $2 \mathrm{keV}$. Le flux moyen d'X était de l'ordre de $2 \times 10^{3} / \mathrm{s} / 0.1 \%$ de la bande spectrale à $2 \mathrm{keV}$ et $6 \times 10^{7}$ à $0.15 \mathrm{keV}$. La brillance moyenne était de $1.5 \times 10^{4} / \mathrm{s} / \mathrm{mrad}^{2} / \mathrm{mm}^{2} / 0.1 \%$ de la BS à $2 \mathrm{keV}$ et $8 \times 10^{4}$ à $0.15 \mathrm{keV}$.
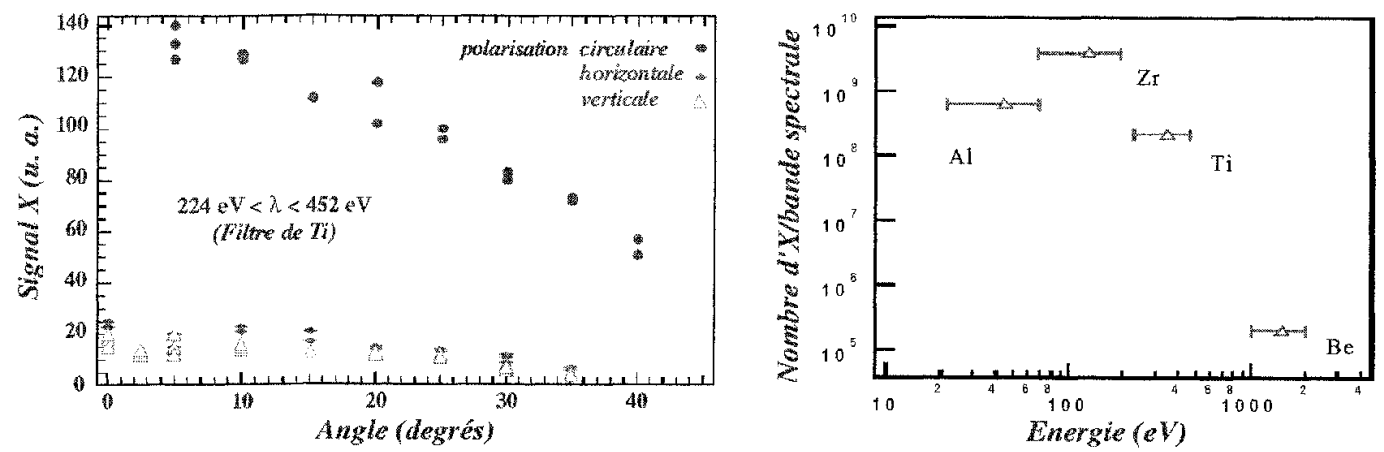

Figure 4. Gauche : distribution angulaire des X d'énergie comprise entre 224 et $452 \mathrm{eV}$. Droite : distribution d'énergie des $\mathrm{X}$ émis suivant l'axe laser. Les barres horizontales correspondent au domaine spectral couvert par chacun des filtres métalliques. Le laser a une polarisation circulaire. Pour ces deux mesures $n_{e}=5 \times 10^{19} \mathrm{~cm}^{-3}$ et la vitesse normalisée d'oscillation des électrons dans le champ laser $\mathrm{v}_{\mathrm{osc}} \mathrm{c}=a_{0} \approx 0.5$.

L'interaction d'une impulsion laser femtoseconde avec un gaz et/ou un plasma peut conduire à de l'émission $X$ via différents processus tels que la génération d'harmoniques, la recombinaison radiative, ou l'émission Bremsttrahlung. Les deux premiers processus sont en contradiction avec l'augmentation du signal en polarisation circulaire et l'observation d'un spectre large et piqué. L'émission Bremsttrahlung peut contribuer au signal $\mathrm{X}$ observé. Cependant aucun de ces trois processus ne peut expliquer l'ensemble des caractéristiques du signal mesuré (présence d'un pic en énergie, croissance linéaire du nombre $\mathrm{X}$ avec $n_{e}$, nombre $\mathrm{d}$ 'X plus élevé en polarisation circulaire, forte sensibilité à l'intensité laser). Seule l'émission par rayonnement de Larmor satisfait toutes ces observations. Ces premiers résultats ont été récemment publiés [6]. Une étude plus détaillée est en cours pour optimiser l'intensité de l'émission Larmor et générer ainsi une source $\mathrm{X}$ polychromatique ultrabrève ( $30 \mathrm{fs}$ ) dans le domaine $\mathrm{du} \mathrm{keV}$.

\section{Remerciements}

Les auteurs remercient J-P. Chambaret, L. Notebaert, M. Pittman, et J-P. Rousseau pour leur aide au cours des expériences menées au Laboratoire d'Optique Appliquée.

\section{Références}

[1] A. Modena et al., Nature 377, 606 (1995).

[2] J. Faure et al., Physics of Plasmas Letters 9, 756 (2002) ; J. Faure et al., Phys. Rev. E 63, 065401(R)

(2001) ; V. Malka et al., Phys. Plasmas 8(6), 2605 (2001).

[3] E.Esarey, P. Sprangle, IEEE Trans. Plasma. Sci. 24, 252 (1996).

[4] J. D. Jackson, Classical Electrodynamics, $3^{\text {rd }}$ Edition (Wiley, New York, 1998).

[5] Y. Ueshima et al., Laser Part. Beams 17, 45 (1999).

[6] K. Ta Phuoc et al., J. Opt. Soc. Am. B 20, 221 (2003). 